Revue

Revue de l'histoire des religions

de Ihistoire des religions

$3 \mid 2020$

Varia

Mathieu BREJON DE LAVERGNÉE, Le temps des cornettes. Histoire des Filles de la Charité, XIX ${ }^{e}-\mathrm{XX}{ }^{e}$ siècle

Paris, Fayard, 2018

Claude Langlois

OpenEdition

Journals

Édition électronique

URL : https://journals.openedition.org/rhr/10791

DOI : 10.4000/rhr.10791

ISSN : 2105-2573

Éditeur

Armand Colin

Édition imprimée

Date de publication : 1 septembre 2020

Pagination : 493-496

ISBN : 978-2-200-93327-2

ISSN : 0035-1423

Référence électronique

Claude Langlois, "Mathieu Brejon de Lavergnée, Le temps des cornettes. Histoire des Filles de la Charité, $x \mid x^{e}-x x^{e}$ siècle », Revue de l'histoire des religions [En ligne], 3 | 2020, mis en ligne le 01 janvier 2021, consulté le 06 janvier 2023. URL : http://journals.openedition.org/rhr/10791 ; DOI : https://doi.org/ $10.4000 /$ rhr. 10791

Ce document a été généré automatiquement le 6 janvier 2023

Tous droits réservés 


\section{Mathieu BREJON DE LAVERGNÉE, Le temps des cornettes. Histoire des Filles de la Charité, $\mathrm{XIX}^{e}-\mathrm{XX}$ siècle}

Paris, Fayard, 2018

\section{Claude Langlois}

\section{RÉFÉRENCE}

Mathieu BREJON DE LAVERGNÉE, Le temps des cornettes. Histoire des Filles de la Charité, XIX XX siècle, Paris, Fayard, 2018, 23,5 cm, 683 p., 35 €, ISBN 978-2-213-709796.

1 Cet ouvrage est important et par le sujet et par la manière de le traiter. La congrégation des Filles de la Charité est en effet la première congrégation active, par l'ancienneté de la fondation qui la rattache directement à saint Vincent de Paul, par le nombre de sœurs et de maisons, par la variété de ses activités, et plus encore par le modèle structurel (la Supérieure générale) largement imité durant tout le XIX ${ }^{\mathrm{e}}$ siècle. L'accès aux riches archives de la Congrégation permet une vision de l'intérieur, à vue surtout des responsables de différents niveaux et de sœurs inspectrices dont les sévérités de jugement étonnent souvent : loi du genre ou bonne connaissance du terrain ?

2 Pour comprendre tout l'intérêt de cette enquête, il convient d'abord d'évoquer les contraintes qui se sont imposées. La première provient du genre "littéraire » dans lequel l'ouvrage s'inscrit, la monographie de congrégation : l'auteur en montre certes la grande plasticité qui lui permet de prendre les libertés nécessaires avec la chronologie, de présenter les supérieures au gré des besoins du récit, de jouer des jeux d'échelle, en alliant centralité des expériences françaises et variété des implantations étrangères. Par contre, il se doit de donner une suite à un premier volume qui avait présenté la famille religieuse de sa fondation au XVIII $^{\mathrm{e}}$ siècle. Le sous-titre de l'ouvrage, de ce fait, ne rend pas exactement compte du temps de cette histoire puisque son $\mathrm{xx}^{\mathrm{e}}$ siècle s'arrête pour l'essentiel à la Seconde Guerre mondiale et que son XIX englobe la Révolution. En 
prenant celle-ci comme point de départ obligé, Brejon de Lavergnée choisit une narrativité qui laisse une grande place à un événementiel souvent dramatique, relayé, après une décennie d'accalmie, par les péripéties d'un schisme durable, contrecoup du conflit qui oppose Napoléon à Pie VII, durant les dernières années de l'Empire. À tout prendre, il aurait peut-être été plus judicieux de nous mener ainsi par la main jusqu'en 1830, en évoquant à la fois les soutiens matériels immédiatement apportés par le Consulat et l'Empire, annualisés dans le budget concordataire, et l'orientation d'une Restauration qui s'intéresse par priorité aux congrégations enseignantes et au modèle conventuel plus traditionnel, dans lequel les filles de la noblesse d'Ancien régime tentent de reprendre pied.

3 L'autre contrainte est celle que l'auteur s'est donné de lui-même : histoire du genre, histoire surtout du care. L'interrogation porte sur les usages qu'il en fait, d'ailleurs inégaux, et sur le déplacement que ces problématiques peuvent introduire dans les manières d'aborder l'histoire d'une congrégation. L'approche en matière de genre, qui implique une attention aux rapports entre les sexes et aux hiérarchies qu'ils construisent, était fort tentante car elle aurait permis de tester la capacité des sœurs à composer avec leurs diverses tutelles masculines (supérieur lazariste, administrateurs des hôpitaux et médecins de plus en plus présents), voire à s'y opposer. En fait, cette approche est davantage suggérée, ici et là, que vraiment analysée. L'ouvrage, et ce n'est pas un mince mérite, concerne avant tout l'histoire des femmes.

4 La réflexion sur le care est par contre expérimentée bien que sur un temps limité (1820-1860). Le care est présenté comme opposable à la charité (p. 149), là où depuis la Révolution, la sécularisation du regard et des pratiques, poursuivie dans la première moitié du XIX ${ }^{e}$ siècle, prônait plutôt de mettre en parallèle la philanthropie avec ladite charité. Mettre en regard la charité avec le care consisterait donc à opposer du théologique, porté par un discours de fondation sans cesse réactualisé, et du philosophique, susceptible de renouveler l'historiographie traditionnelle. La définition, maintenant classique, du care, unissant sollicitude (de la part du sujet) et soin au bénéfice de personnes vulnérables, dans la quotidienneté du geste, est légitimement revendiquée (p.150). L'auteur qui, dans ces derniers chapitres, nous introduit justement sur le terrain d'un soin multiforme, nous oblige à prendre au sérieux ce questionnement mais aussi à nous interroger sur son applicabilité : le care ne pourraitil pas nous permettre de mieux historiciser le périmètre que la société et la congrégation donnent aux sujets vulnérables? En reprenant la réflexion initiale sur le rapport entre care et charité, ne mettrait-on pas mieux en évidence ce qui fonde la réactivité de la congrégation à toutes les sollicitations qui lui sont faites tout en nous obligeant à nous demander ce que devenait cette pratique au fil des décennies? On pourrait peut-être alors lui imputer le peu d'attention portée par la congrégation à l'institutionnalisation d'un enseignement primaire auquel celles et ceux qui se réclament de la postérité lassalienne sont davantage préparés à faire face. Il faudrait surtout montrer comment les Filles de la Charité se sont trouvées prises dans un processus de fonctionnarisation comme cadres dans le secteur hospitalier, alors que les congrégations nouvelles inventent de nouvelles modalités d'un soin direct, comme les Petites sœurs des pauvres qui partagent la vie des vieillards ou les garde-malades à domicile qui veillent longuement sur les malades dans des cadres familiaux disparates.

5 Toutefois ce que cet ouvrage perd dans ses marges historiques ou réflexives, il le gagne largement par ce qu'il apporte en se concentrant sur l'identification et le 
fonctionnement de la plus grande internationale de la charité et sur l'identité toujours difficile à maintenir et à renouveler à la tradition vincentienne à travers l'espace et le temps. C'est bien à cela qu'est consacré le cœur même de l'ouvrage (chapitres V à XIII), en jouant avec une grande habileté sur plusieurs registres, la réactivité à la demande de la société française et aux contraintes de la politique, les modalités de l'action internationale, l'attention plus particulière à l'activité emblématique des Filles de saint Vincent de Paul, les orphelinats ou à la spiritualité à travers la poussée mariale du xIx ${ }^{e}$ siècle, dossier qui aurait pu être mieux incorporé dans l'histoire même de la congrégation. De ce fait cette enquête fort bien documentée et clairement présentée va au-delà de ce qu'elle apprend sur la réactivité de la congrégation à l'interdiction d'enseigner en France (1904) ou sur l'entrée dans la professionnalisation hospitalière, pour ne prendre que deux chapitres fort neufs.

Ce qui, à mon sens, fait l'extrême intérêt de cet ouvrage, c'est cela même qu'il révèle, la constitution d'un grand siècle (1830-1940) qui sans doute aurait pu se prolonger sur une voire deux décennies: cette étude met en évidence le temps d'une congrégation redevenue dynamique et conquérante qui, entre les deux guerres, compte 38000 sœurs de par le monde, majoritairement européennes, mais où les Françaises, devenues minoritaires continuent à fournir les cadres d'une entreprise caritative qui trouve en sœur Inchelin (1861-1940), infirmière devenue Supérieure générale en 1922, une personnalité d'exception pour diriger la congrégation en des temps difficiles.

Dans ce temps actuel où l'Église se trouve en procès à la fois historique (le temps et les modalités de son effondrement) et médiatique (la gestion calamiteuse de la pédophilie cléricale), cet ouvrage offre des éléments positifs susceptibles de porter sur l'institution un jugement plus équilibré et peut-être même fournir des éléments d'une nouvelle apologétique, mais il est avant tout un probe travail d'historien. À ce titre, il incite, à partir de l'objet même de son étude, à opérer un triple déplacement du regard. Par l'intérêt d'abord porté au temps long d'une visibilité catholique que le titre - Le temps des cornettes - rappelle avec humour, temps qui, dans le catholicisme, ne peut se réduire à celui de la pratique des fidèles ou des conflits théologiques voire idéologiques: le croire et le faire catholiques passent en effet par ces chemins dont il fallait rappeler la durable importance. Par la mise en évidence en conséquence d'un catholicisme d'œuvres dont la plasticité est aussi grande dans l'action même des sœurs que dans celle de son public laïc, des Enfants de Marie aux premières syndicalistes de la CFTC. Par la visibilité enfin d'un catholicisme au féminin qui est alors à son apogée et s'exporte largement hors de France. Sur ce dernier point, on pourrait sans doute poursuivre la réflexion en se demandant comment peut s'articuler un tel activisme caritatif avec une mystique féminine renaissante durant la même période, autour de la figure de Thérèse de l'Enfant Jésus. On pourrait aussi s'interroger sur les limites d'une féminisation du catholicisme qui s'est finalement heurtée à la sécularisation de la société après 1950 mais aussi à l'intangibilité des structures du catholicisme qui, à tous les niveaux, place toujours la totalité des pouvoirs entre les mains d'un clergé masculin bien en mal de célibat. 


\section{AUTEURS}

\section{CLAUDE LANGLOIS}

École Pratique des Hautes Études. 